\title{
Unicast Routing on VANETs
}

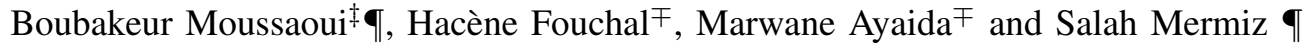 \\ ¥ Laboratoire D'électronique et des Télécommunications Avancées (ETA), \\ Université Mohamed Bachir El Ibrahimi de Bordj Bou Arreridj, 34031, Algeria \\ F Centre de recherche CReSTIC, \\ Université de Reims Champagne-Ardenne \\ 51687 REIMS Cedex 2, France \\ I Université de Constantine -Abdelhamid Mehri- Algeria \\ Moussaoui.bkr@gmail.com, \{hacene.fouchal, marwane.ayaida \}@univ-reims.fr, s_merniz@hotmail.com
}

\begin{abstract}
Greedy routing in VANETs requires some geographical informations, such as the source location and the destination location. The first one could be obtained using some localization devices like GPS receiver. However, the second one is provided by a location service. This later has a high overhead especially if it is implemented over V2V (vehicle to vehicle) communications. Many location services are well known as HLS, RLS, GLS.

This paper is interested in reducing this overhead by using some Road Side Units (RSU) already deployed along the roads. We propose here a location service called "improved Reactive Location Service (iRLS)", which is an extension of the RLS service. The major difference is that RLS assumes only V2V communications and iRLS takes profit of a wireless backbone based on RSUs to catch the destination's position. This allows to reduce the overhead instead of flooding requests and also makes the communication faster since we will not have to wait to the request to reach the destination before receiving the response and then starting sending data. In our proposal, the closest RSU will reply with the actual location.

In order to show the contribution of our approach, we have conducted some simulations that prove that iRLS outperforms any geographic protocol by using the V2I communications in terms of end-to-end delay which is one of the most important parameter. We considered also the ratio of packet received correctly by the destination vehicle (PDR), our protocol improves significantly this second parameter, and ensures more than $20 \%$ of packets received correctly

Index Terms-VANETs; Location-based Services; Geographic
\end{abstract} Routing Protocols, .

\section{INTRODUCTION}

$\mathbf{V}$ ANETs (Vehicular Ad-hoc NETworks) are a special case of MANETs (Mobile Ad-hoc NETworks). Therefore, the routing protocols used for MANETs could be adapted for MANETs. However, the topology-based protocols in MANETs are not useful for VANETs because of the high mobility rate. Then, geographic routing protocols are more suitable for such networks since they are more scalable.

The geographic routing protocols need as an input the node's location as well as the destination's location. The node's position is easy to obtain. However the destination's position is obtained from a location service. This service is responsible to reply to requests like : "Where is the node X?". "Reactive Location Service" (RLS) is a well known location service. When a node needs to find the position of another node, it floods a request. When this request reaches the destination, this latter replies with its current position using the same path for the request delivery. Therefore, RLS uses only V2V communication without considering the V2I (vehicle to infrastructure) ones. For this purpose, we propose a new location service denoted "improved Reactive Location Service" (iRLS). This service uses mainly the RSU network to reply directly to the location request in order to avoid previous flooding. This allows to gain in term of overhead and delay as demonstrated by the simulations that have been conducted using the well known NS-3 simulator.

The paper is organized as follows. The section II presents some works related to the location-based service and the geographic routing protocols. Section III described our iRLS proposal. Section IV introduces the simulations conducted with our proposal. Finally, section V concludes the paper and presents some future works.

\section{RELATED WORKS}

In this section, we will describe some related works, published these last two decades. All these works focused on routing in VANET environments where most of solutions used V2V communications and/or V2I communications. They do not consider position changes during the dissemination process. Both the source node and the destination node are vehicles in general case. To deal with the high moving speed and dynamic changes of the topology, RSUs can provide a lot of benefits in order to achieve efficient routing of messages over the network. In order to propose a realistic solution,, we do not need to deploy an RSU at each road intersection. An overview on routing for mobile and vehicular ad hoc networks is well detailed in [1].

In [2], authors propose an improved version of DGRP (Directional Greedy Routing Protocol). In addition to the information used by DGRP (position, speed, direction) provided by a GPS sensor, they consider and evaluate link stability. As a result, they reduce links breakage, enhance reliability of a used path and ensure a high packet delivery ratio.

Another proposed work which assists routing in VANETs using base station was presented in [3]. Roads are composed of segments, each of them is governed by a base station. This later sends a response to a route request packet by choosing the shortest path using RSUs. But if we do not 
have any available path, the RSU checks if there is enough free bandwidth to grant the request. They have used two mechanisms, using Fast Learning Neural Networks, to prevent link failure and to predict bandwidth consumption during handoffs. An alternative path will be established, before that a broken link occurs.

Authors in [4] have proposed an infrastructure-assisted routing protocol. The main benefits behind this approach is to reduce the routing overhead and improve the end-to-end performance. A backbone network ensures connectivity by using RSUs, an enhancing search of the shortest path to reach the destination is done by this infrastructure. Authors present an extended protocol to the topology-aware GRS routing protocol. At any intersection, anchor nodes in the GRS system compute the shortest path to reach the destination using the Djikstra algorithm without considering the road density or any other parameter which can ensure connectivity of roads.

Roadside-Aided Routing (RAR) has been proposed in [5]. It is another study which prefers V2I communication to improve the search for a stable routes over VANETs networks.

\section{A. Location-based services}

Location-based services can be classified into two classes : "Flooding-based" and "Rendez-vous-based". The first class is composed of reactive and proactive services. In the proactive flooding-based location-based service, every node floods its geographic information through all the network periodically. Thus, all the nodes are able to update their location tables. Since this approach uses flooding and may surcharge the network by location update messages, several techniques to reduce the congestion were used. One of them is to tune the update frequency with the node mobility (the more node is moving fast, the higher update location frequency is used).

Therefore, the update frequency decreases with the distance to the node. The second idea is, a node with high mobility sends more update location packets. As a result, there are less packets than a simple flooding scheme without affecting the network performances. For the second group (i.e the reactive flooding-based location-based service), the location response is sent when receiving a location request. This avoids the overhead of useless location information of some nodes updated and never used. But, it adds high latencies not suitable in VANETs. One of these known services is Reactive Location Service (RLS) [6].

In the second class (rendez-vous-based location service), all the nodes agree on a unique mapping of a node to other specific nodes. The geographic information are disseminated through the elected nodes called the "location servers".

Thus, the location-based services consists of two components :

1) Location Update : A node has to recruit location servers (chosen from other nodes) and needs to update its location through theses servers. The location servers are responsible of storing the geographic data of the relating nodes.
2) Location Request : When a node needs to know the location of another node, it broadcasts a location request. The location server will replay as soon as it receives this request.

\section{B. Geographic Routing Protocols}

Routing protocols algorithms must choose some criteria to make routing decisions, for instance the number of hops, latency, transmission power, bandwidth, etc. The topology-based routing protocols suffer from heavy discovery and maintenance phases, lack of scalability and high mobility effects (short links). Although, geographic routing are suitable for large scale dynamic networks. The first routing protocol using the geographic information is the Location-Aided Routing (LAR) [7]. This protocol used the geographic information in the route discovery. This latter is initiated in a Request Zone. If the request doesn't succeed, it initiates another request with a larger Request Zone and the decision is made on a routing table. The first real geographic routing protocol is the Greedy Perimeter Stateless Routing (GPSR) [8]. It is a reactive protocol which forwards the packet to the target's nearest neighbor (Greedy Forwarding approach) until reaching the destination. Therefore, it scales better than the topologybased protocols, but it does still not consider the urban streets topology and the existence of obstacles to radio transmissions. Another geographic routing protocol is the Geographic Source Routing (GSR) [9]. It combines geographical information and urban topology (street awareness). The sender calculates the shorter path (using Djikstra algorithm) to the destination from a map location information. Then, it selects a sequence of intersections (anchor-based) by which the data packet has to travel, thus forming the shortest path routing. To send messages from one intersection to another, it uses the greedy forwarding approach. The choice of intersections is fixed and does not consider the spatial and temporal traffic variations. Therefore, it increases the risk of choosing streets where the connectivity is not guaranteed and losing packets.

In [10], authors propose an improved version of DGRP (Directional Greedy Routing Protocol). In addition to the information used by DGPR (position, speed, direction) provided by GPS, they consider and evaluate link stability. As a result, they reduce links breakage, enhance reliability of a used path and ensure a high packet delivery ratio.

\section{IMPROVED LOCATION-BASED SERVICE}

To better understand our proposal, we present a simple scenario in the Figure 1. We suppose that the vehicle V1 has data to send to the vehicle V6, which is not in its direct neighborhood. If $\mathrm{V} 1$ has a valid and fresh route in its routing table, it sends immediately the data without any routing service requirement, otherwise it broadcast a request to find a suitable route to use. Since V1 is in the RSU4's range, V1 sends a request to RSU4 asking for the new V6's location. RSUs are connected together and exchange vehicles' positions when needed. After receiving the response from RSU4, V1 starts sending data using the greedy approach, where it selects 
the closest neighbor to the destination V6 (here V8). Then, data packets travel through V8, V7 and finally to reach V6. Therefore, destination's location could be found more rapidly than using $\mathrm{V} 2 \mathrm{~V}$ communications when a destination node is far away from the source node;

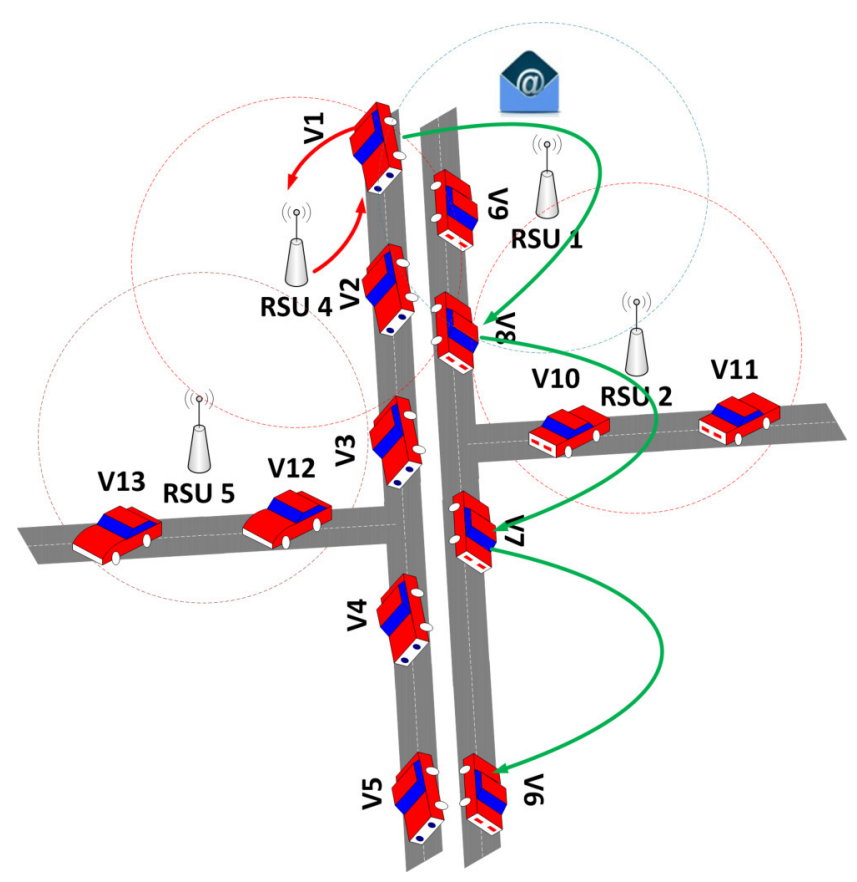

Figure 1. An example of a scenario with iRLS

The algorithm 1 presents more details how the iRLS service works. When starting, the source $s$ looks if it is not in the range of a RSU, then it works like RLS. It floods a request to find the location of the destination $d$. When the request reaches the node $\mathrm{Z}$ that has this information, the latter replies directly to $s$ with d's new position. Otherwise, the node $s$ sends the request in unicast way to this RSU (RSUX). RSUX forwards this request to all connected RSUs. When RSUY receives the request and it has already this information, it replies to RSUX. RSUX then transmits the d's position to node $s$. Therefore, $s$ has all required information to start sending data. These data are forwarded using the greedy approach until reaching the destination. By reducing the $\mathrm{V} 2 \mathrm{~V}$ search phase, the available limited bandwidth of our links will be used to transmit the effective data rather than the transmission of control packets. Indeed, our protocol ensures a higher PDR than the standard protocol (see Figure 3).

In the case where a node $S$ has no RSU in its neighborhood, it first sends a request to find the closest RSU. This request is run in a setup step before starting the routing process.

But in some cases, there is not enough vehicle density and the initial request could not be sent (caused by a lack of connectivity). We suggest to resend the packet after a defined time (timeout) until a reply is received from a close RSU.

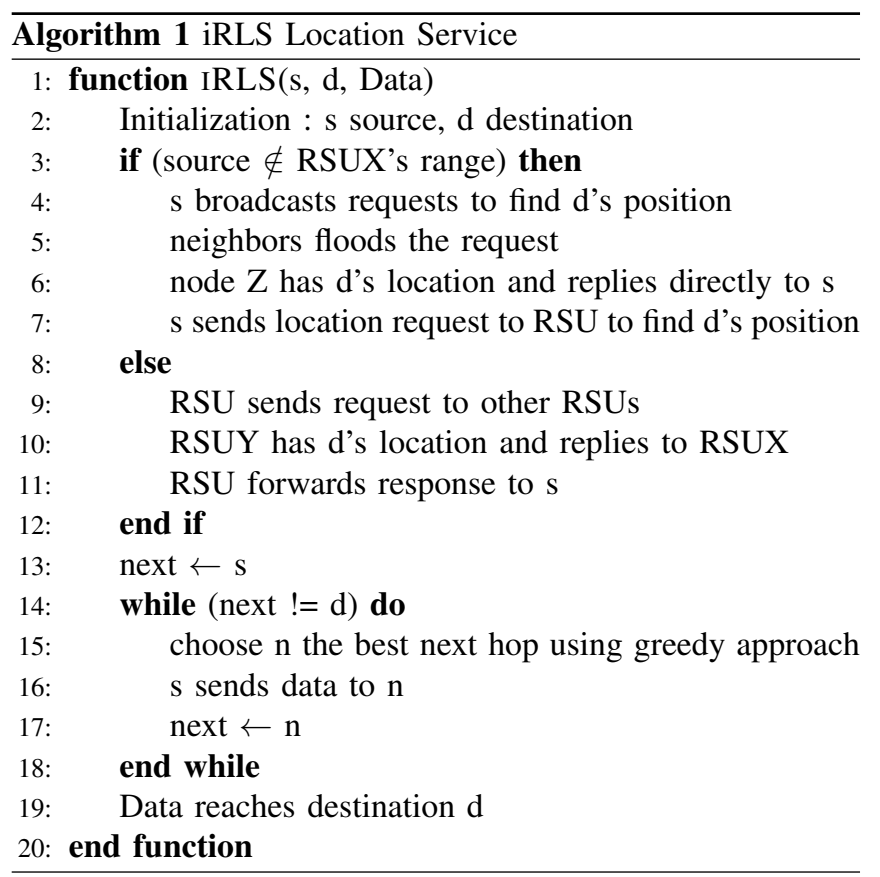

\section{Simulations}

\section{A. Working Environment}

The simulations were performed using the Ns-2 simulator 2.33 [11]. The geographic routing protocol used is the Greedy Perimeter Stateless Routing (GPSR) [8]. The chosen area is a $2 \times 2 \mathrm{~km}^{2}$ of a real map representing part of the French city Reims. This area is extracted from Open Street Map [12]. The MAC layer used is $802.11 \mathrm{p}$ [13]. The parameters used in the simulation are summarized in the Tab. I.

At each simulation, every node initiates $4 \mathrm{CBR}$ traffics of 100 packets with a size of $128 \mathrm{~KB}$ to 4 random destination nodes with a second of interval between each sent message. The CBR traffic simulates for example an audio or a video streaming. It may be used in security applications, such as viewing the video stream from a camera located on a bus by the police car or the security agent vehicle. Also, this traffic could be used in entertainment applications to connect to the Internet or to play online video games.

\section{B. Experimentation Results}

Our experimentations have provided the following figures. On each figure, we show the network behavior with usual RLS (denoted RLS curve) and the behavior of our proposed solution (denoted iRLS curve for improved RLS). Figure 2 shows the delay to send a message depending on the size of the network from 10 nodes to 100 nodes. we observe that the delay to send a message from a node $s$ to $d$ decreases with iRLS even if the number of nodes is higher. The gain is around 10 per cent. In Figure 3, the results highlight that our protocol can achieve a higher PDR than the RLS one. In the density 100 vehicles, it is clear that taking the same scenario of simulation, our protocol guarantee the same ratio but when using RLS this ratio decreases. 
Table I

THE SIMULATION PARAMETERS

\begin{tabular}{|c|c|}
\hline Parameters & Value \\
\hline Channel type & Channel/WirelessChannel \\
\hline Propagation model & Propagation/TwoRayGround \\
\hline Network interface & Phy/WirelessPhyExt \\
\hline MAC layer & $802.11 \mathrm{p} \mathrm{[13]}$ \\
\hline Interface queue type & Queue/DropTail/PriQueue \\
\hline Link layer & LL \\
\hline Antenna model & Antenna/OmniAntenna \\
\hline Interface queue length & 512 packets \\
\hline Ad-hoc routing protocol & GPSR \\
\hline Location-based service & RLS \\
\hline Location cache maximum age & $4,8,12,16$, and $22 \mathrm{~s}$ \\
\hline Area & $2 \times 2 \mathrm{~km}^{2}$ \\
\hline Number of nodes & $10-150$ \\
\hline Simulation time & $150 \mathrm{~s}$ \\
\hline GPRS beacon interval & $0,5 \mathrm{~s}$ \\
\hline CBR traffic & $4 \times 100$ packets / node \\
\hline CBR packet size & $128 \mathrm{~KB}$ \\
\hline CBR sent interval & $1 \mathrm{~s}$ \\
\hline
\end{tabular}

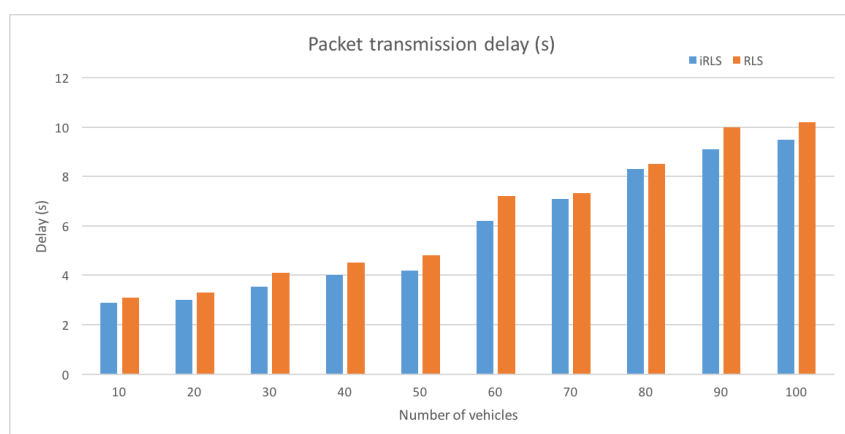

Figure 2. The average delay for packet transmission

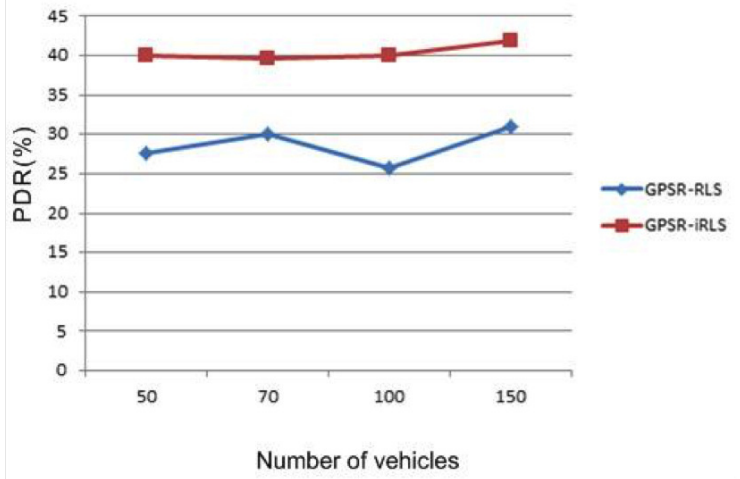

Figure 3. Impact of the number of vehicles on the achieved PDR average

These results show that our protocol outperform by more than $10 \%$ in general case the use of RLS. All these result can be explained by the best consumption of the network resources like bandwidth. This observed result is quite predictable since the RLS process uses the V2V communications (a RSU is considered as a vehicle also) then the request is sent through the whole network without any efficiency. Contrary to RLS, iRLS takes advantage of the RSU backbone which provide a fast connection between the initial RSU and the closest to the destination one.

\section{CONCLUSION \& FUTURE WORKS}

This paper presents the iRLS location service. This location service takes advantage of deployed RSUs on roads in order to ensure fast replies of destination lookup . RSUs are already wirelessly connected. Therefore, they can exchange data of the nodes position. This enhances the network routing performances. As a consequence, network performances such as the end-to-end delay are better than in the original RLS service. The presented work is realistic since it is applicable in any deployed C-ITS (cooperative Intelligent Transport System) and offers the ability to route packet from a node to another with interesting performances.

As future works, we intend to use the RSUs backbone to send real data. We intend also to study the scalability of such a mechanism. It could be interesting also to evaluate our protocol with another kind of protocols (Topology based protocol), with bandwidth metrics.

\section{ACKNOWLEDGEMENT}

This work is partially supported by the EC SCOOP project (INEA/CEF/TRAN/A2014/1042281).

\section{REFERENCES}

[1] R. Rana, S. Rana and K. C. Purohit, "A review of various routing protocols in VANET," International Journal of Computer Applications, vol. 96-18, 2014.

[2] K. Prasanth, D. K. Duraiswamy, K. Jayasudha and D. C. Chandrasekar, "Improved packet forwarding approach in Vehicular ad hoc networks using RDGR algorithm", arXiv preprint arXiv:1003.5437, 2010.

[3] C. J. Huang, Y. T. Chuang, . Y. J. Chen, D. X. Yang and I.F Chen, "QOSaware roadside base station assisted routing in vehicular networks." Engineering Applications of Artificial Intelligence, 22(8), $1292-1301$

[4] G. J. Borsetti, "Infrastructure-assisted geo-routing for cooperative vehicular networks," IEEE Vehicular networking conference (VNC), 2010, pp 255-262.

[5] Y. Peng, Z. Abichar and J.M. Chang, "Roadside-aided routing (RAR) in vehicular networks." IEEE International Conference on ICC'06.

[6] Käsemann, M., Füßler, H., Hartenstein, H., Mauve, M. (2002). *A reactive location service for mobile ad hoc networks*. Universität Mannheim/Institut für Informatik, Technical report REIHE INFORMATIK 14/2002. 14/2002

[7] Y.-B. Ko and N. H. Vaidya, "Location-aided routing (LAR) in mobile ad hoc networks," Wireless Networks, vol. 6, no. 4, pp. 307-321, Jul 2000.

[8] B. Karp and H. T. Kung, "GPSR: Greedy Perimeter Stateless Routing for Wireless Networks," in Proceedings of the 6th annual international conference on Mobile computing and networking (MobiCom'00), New York, NY, USA, 2000, pp. 243-254.

[9] C. Lochert, H. Hartenstein, J. Tian, H. Fuessler, D. Hermann, and M. Mauve, "A routing strategy for vehicular ad hoc networks in city environments," in In Proceedings of the IEEE Intelligent Vehicles Symposium, 2003, pp. 156-161.

[10] K. Prasanth, D. K. Duraiswamy, K. Jayasudha, and D. C. Chandrasekar, "Improved packet forwarding approach in vehicular ad hoc networks using rdgr algorithm," arXiv preprint arXiv:1003.5437, 2010.

[11] NS2 : Network Simulator. [Online]. Available: http://nsnam.isi.edu/ nsnam/

[12] OpenStreetMap. [Online]. Available: http://openstreetmap.fr/

[13] NS2 802.11p implementation. [Online]. Available: http://dsn.tm. uni-karlsruhe.de/english/Overhaul_NS-2.php/ 